\title{
Programa de Educación Universitaria para jóvenes que presten servicio militar en zonas de frontera - Perú UniFFAAGoL
}

\author{
University Education Program for young people who serve in the military in border areas - Perú UniFFAAGoL \\ Programa de Educação Universitária para jovens servindo nas forças armadas em áreas de \\ fronteira - Peru UniFFAAGoL
}

$\begin{array}{rr}\text { Carlos A. Bocanegra García } & \text { Freddy M. Camacho Delgado } \\ \text { cbocanegra@unitru.edu.pe } & \text { fmcamacho@unibagua.edu.pe } \\ \text { https://orcid.org/0000-0001-9063-7600 } & \text { https://orcid.org/0000-0002-3053-5300 } \\ \text { Universidad Nacional Intercultural } \\ \text { Universidad Nacional de Trujillo, Trujillo-Perú } & \text { Fabiola Salazar Leguía, Bagua-Perú }\end{array}$

Artículo recibido en junio 2021, revisado en julio 2021, arbitrado en agosto 2021 y publicado en septiembre 2021

\section{RESUMEN}

Se presenta un Programa de educación universitaria inclusiva para jóvenes que presten servicio militar en zonas de frontera, al cual se le puede denominar UniFFAAGoL, sustentado en los actores: Universidad, Fuerzas Armadas (FFAA), y Gobiernos Locales. El objetivo fue contribuir al desarrollo local utilizando sus fortalezas. El programa es una respuesta a la falta de oportunidades educativas a nivel superior en las zonas de frontera, al escaso desarrollo de las comunidades, y poco interés por el servicio militar voluntario, la creciente desocupación y con ello la delincuencia. La propuesta surge ante la pregunta como enfrentamos creativamente o desarrollamos la "imaginación" para superar esta situación. La investigación se desarrolló empleando el método etnográfico. Los resultados demuestran la factibilidad del programa y puede ser un referente bajo las condiciones descritas.

Palabras clave: Educación universitaria; Servicio militar; Zonas de frontera; Desarrollo Local; Universidad inclusiva

\section{ABSTRACT}

An inclusive university education program for young people who perform military service in border areas is presented, which can be called UniFFAAGoL, supported by the actors: University, Armed Forces (FFAA), and Local Governments. The objective was to contribute to local development using its strengths. The program is a response to the lack of educational opportunities at the higher level in the border areas, the scarce development of the communities, and little interest in voluntary military service, the growing unemployment and with it the delinquency. The proposal arises from the question of how we creatively face or develop the "imagination" to overcome this situation. The research was carried out using the ethnographic method. The results demonstrate the feasibility of the program and can be a benchmark under the conditions described.

Key words: University education; Military service; Border zones; Local development; Inclusive university

\section{RESUMO}

É apresentado um programa de educação universitária inclusiva para jovens que prestam serviço militar em áreas de fronteira, que pode ser denominado UniFFAAGoL, apoiado pelos atores: Universidade, Forças Armadas (FFAA) e Governos Locais. O objetivo era contribuir para o desenvolvimento local usando seus pontos fortes. $\mathrm{O}$ programa é uma resposta à falta de oportunidades educacionais de nível superior nas áreas de fronteira, ao escasso desenvolvimento das comunidades e ao pouco interesse pelo serviço militar voluntário, ao crescente desemprego e com ele à delinquência. A proposta surge da questão de como enfrentamos criativamente ou desenvolvemos a “imaginação" para superar esta situação. A pesquisa foi realizada por meio do método etnográfico. Os resultados demonstram a viabilidade do programa e podem servir de referência nas condições descritas.

Palavras-chave: Educação universitária; Serviço militar, Zonas fronteiriças, Desenvolvimento local, Universidade inclusiva 


\section{INTRODUCCIÓN}

A nivel nacional, se reconoce que los pueblos originarios deben hacer frente a múltiples desafíos en el ámbito de la educación. Vivimos en un mundo cada vez más multicultural y la concepción tradicional del contenido de los planes de estudio y de los métodos de enseñanza, incluso en aquellos estados cuya organización se basa en una supuesta homogeneidad cultural (Rodolfo Stavenhagen ha acuñado el calificativo de "etnocráticos" para ese tipo de estados) sencillamente ya no funciona. La incertidumbre nunca ha sido mayor en el mundo, como han señalado tanto la Comisión Mundial de Cultura y Desarrollo (Comisión Pérez de Cuéllar) como la Comisión Internacional sobre la Educación para el siglo XXI (Comisión Delors). Además, en la mayoría de los países, casi no se toma en cuenta a los pueblos y minorías indígenas a la hora de definir los planes de estudio y de organizar la enseñanza. De hecho, en muchos países los principios básicos de educación multicultural y multilingüe establecidos por la UNESCO (2004) apenas se han aplicado.

Constituye una tendencia desarrollar la educación con programas inclusivos como el propuesto por la UNESCO (2019), para con los niños migrantes y refugiados, al privarlos de sus derechos y hacer caso omiso de sus necesidades. Los migrantes, los refugiados y las personas internamente desplazadas figuran entre los grupos de población más vulnerables del mundo, e incluyen a quienes viven en tugurios, a los que se desplazan según las estaciones para poder ganarse la vida, $y$ a los menores detenidos. Sin embargo, se les niega a menudo rotundamente el acceso a las escuelas, que son para ellos un refugio seguro y entrañan la promesa de un futuro mejor.

Es evidente que cada pueblo originario o indígena desarrolla y requiere de programas de educación tan diversa como sus culturas y sociedades donde viven. Este hecho ha implicado que en la actualidad, exista en todo el mundo una vasta gama de enfoques y programas educativos destinados a esos pueblos. Es importante mencionar que no se trata de crear universidades para cada grupo cultural, sino de asumir la interculturalidad desde el enfoque de la educación multicultural. En este sentido son válidas los argumentos de Linda King y Sabine Schielmann (2004), que dicen que la expresión "educación indígena" no se refiere a un tipo de educación diferente, exclusivo o paralelo, sino que hace hincapié en la necesidad de satisfacer las necesidades específicas de los pueblos indígenas a fin de suscitar una toma de conciencia y ampliar el debate con vistas a lograr una educación. Agregaría dar oportunidades en todo el sistema educativo nacional y en especial en las zonas de frontera donde las necesidades son mayores.

Es innegable que actualmente existe una brecha de recursos humanos calificados en las zonas de frontera, que limita la capacidad del sistema educativo, de salud, de defensa. Es notoria la carencia de profesionales en salud, nutrición, tecnológica y de administración Sin embargo se cuenta con programas como Beca 18 (PRONABEC, 2016) y otras modalidades de becas, que corresponden cada una a un objetivo específico y / o interés nacional específico. Unos objetivos responden a focalizaciones de lugares que por razones históricas o sociales fueron priorizadas (Huallaga, VRAEM). Asimismo, se habla de vulnerabilidad por mi experiencia universitaria en Amazonas, puedo manifestar que Beca 18 en términos generales aporta con la formación de los jóvenes originarios y pobres, pero que los que se forman en las grandes ciudades y en particular en Lima, no garantiza su retorno a sus pueblos originarios y con ello no se cumple el objetivo de desarrollar los pueblos vulnerables y poco atendidos. 
También se cuenta con el Programa de Entrenamiento en Salud Pública dirigido a jóvenes que realizan el servicio militar acuartelado. El programa consta de siete unidades temáticas dictadas en 390 horas, de las cuales 200 corresponden a prácticas de aulas y de campo. Entre julio de 2014 y diciembre de 2015, el programa se ha desarrollado en diez sedes de las tres fuerzas armadas, capacitando y certificando a 405 jóvenes. La intervención brinda un beneficio educativo adicional al servicio militar, haciéndolo más atractivo como oportunidad de desarrollo y abre una línea laboral en el campo de la salud pública a jóvenes de las zonas más alejadas (Ministerio de Salud, 2019). Esta experiencia busca incrementar los recursos humanos entrenados en salud pública a nivel técnico operativo. En coordinación con los gobiernos regionales se espera que los egresados sean acogidos e insertados en el sistema de salud pública. Sin embargo, continúa siendo insatisfecha las expectativas de los jóvenes que prestan servicio militar, pues al término de su servicio no tienen mayores oportunidades que enrolarse a las actividades rurales de donde provienen, incrementar la desocupación y los vicios de ella se derivan.

Actualmente asistimos a una serie de opiniones sobre la pertinencia o no de la Ley que aprueba el llamamiento extraordinario para la incorporación al servicio militar, en caso no se logre alcanzar el número necesario en el llamamiento ordinario que anualmente convoca el Ministerio de Defensa (2007) con el propósito de cubrir las necesidades de personal militar acuartelado. Según el artículo 50 de la Ley del servicio militar, modificado por el Decreto Legislativo 1146, establece la obligatoriedad para quienes salgan sorteados. El fundamento que justifica dicha obligatoriedad es el déficit de $20 \mathrm{mil}$ plazas anuales, vale decir no se cubre las necesidades de personal militar. Por otro lado, la universidad se ha caracterizado por ser centralista y excluyente, que a pesar de iniciativas como el Programa Beca 18 no alcanza a cumplir con el objetivo de desarrollar las comunidades de donde proceden los egresados pues en su mayoría quienes son formados en las grandes ciudades terminan por no regresar a sus pueblos originarios y no aportan significativamente al desarrollo nacional. Es una necesidad imperiosa que la nueva universidad y dentro de ellas las interculturales y de frontera desarrollen modelos de gestión universitaria, que de cara a los retos de su entorno y de la globalización, que contribuya efectivamente al desarrollo y la paz. Por ejemplo, si hablamos de una universidad asentada en la zona andina o en la selva sobre todo en zona de frontera , ésta debe considerar además de generación de conocimiento, calidad educativa, procesos de internacionalización, mayor competitividad, el acercamiento a las comunidades alejadas que poseen talentos humanos que por siempre han estado excluidas de la oferta académica, y una de ellas es precisamente incorporar a los jóvenes que voluntariamente cumplan con el servicio militar como uno de los beneficios al esfuerzo de la defensa de la patria.

En este marco las universidades de frontera, tienen la oportunidad de convertirse en el eje del desarrollo local, en alianza con los Municipios (Gobiernos Locales) y las Fuerzas Armadas (FFAA). Este modelo de inclusión he tenido la oportunidad de ponerlo a prueba en la Universidad de la Amazonia Mario Peláez Bazán (Utcubamba - Amazonas, ahora Universidad de la Amazonía), donde 5 jóvenes de la región Amazonas, paralelamente prestaban servicio militar no acuartelado, asumían el pago de las pensiones o derechos de enseñanza parcialmente con las propinas que les otorgaba el ejército peruano, que además les da no solo facilidades para estudiar sino que también les 
otorga alimentación y alojamiento. En esta misma universidad logramos vía convenios de compromiso municipal a la formación profesional de jóvenes de su jurisdicción, con becas a 20 jóvenes formándose para luego contribuir al desarrollo de sus pueblos. Por la experiencia académica y profesional hasta ahora desempeñada, me permito plantear algunos aspectos que justificarían el Programa UniFFAAGoL, como alternativa a la demanda insatisfecha de jóvenes voluntarios para el servicio militar, paliar la desocupación y la delincuencia (Bocanegra, 2012, 2013).

Es común constatar que no existen profesionales de la localidad en la mayoría de los pueblos del interior de los Departamentos de frontera y los pocos que existen solo permanecen por días, pero lo más lamentable es que las plazas para profesionales en enfermería por ejemplo, no se cubren en los pueblos fronterizos, el caso más representativo se presenta en la Provincia de Condorcanqui (Amazonas), donde es diario observar la falta de atención, abandono y vulnerabilidad de la población por su condición de pobreza y pobreza extrema, hecho que se agudiza con la pandemia del Covid-19.

Se conoce que las zonas de frontera son espacios sometidos a tensiones generadas por los procesos migratorios que en el último año se ha intensificado por el masivo ingreso de pobladores de Venezuela. Pero además en estas zonas, viven poblaciones originarias principalmente que se caracterizan por altos índices de pobreza, desnutrición y falta de oportunidades de desarrollo individual y colectivo. Abordar el estudio de las zonas fronterizas implica casi siempre un desafío puesto que se trata de espacios de reciente configuración en la historia de América Latina, trazados en zonas de baja densidad, alejadas de los centros políticos y definidas como áreas aisladas y remotas Amilhat-Szary y Rouviére (2009).
La noción de frontera es ampliamente utilizada en las ciencias sociales con fines distintos $\mathrm{y}$ significados diversos, $\mathrm{y}$ en el último tiempo aparece frecuentemente citada en los estudios sobre globalización (Zapata-Barrero y Ferrer-Gallardo 2012). Pero de lo que se trata es de la articulación entre frontera, oportunidad de educación superior, mercado laboral, patrones migratorios y de movilidad fronteriza constituyen patrones a tomar en cuenta si de desarrollo y defensa de la frontera se trata. La idea de la defensa de la frontera como recurso pasa por generar oportunidades para sus habitantes que a la fecha son invisibles en el ámbito educativo y de la salud principalmente.

En un contexto de zonas de frontera caracterizados por desigualdades de desarrollo, agravado por la marginalidad social especialmente en el caso de los departamentos fronterizos de la amazonia, la posibilidad de los jóvenes de involucrarse en objetivos ilegales es muy alta, hecho que ha convertido a las zonas de frontera en un espacio de circulación y de actividades en su mayoría ilícitas, con mercados laborales precarizados , mano de obra barata y flexible con quienes están "dispuestos" a aceptar ciertas condiciones de trabajo. Dificultad, porque en muchos casos la cruzan a pie $\mathrm{y}$ van y vuelven, porque la frontera históricamente no ha sido concebida como una barrera.

Si analizamos los niveles de pobreza para el caso particular de Amazonas, ésta cuenta con 10 distritos ubicados entre los 150 más pobres de Perú, con una tasa de pobreza que oscila entre el $65,7 \%$ y el 91.7 $\%$. Uno ubicado en la provincia de Condorxanqui (El Cenepa), cinco en Luya (Pisuquia, Santa catalina, San jerónimo, Lónguita y Cocabamba), tres en Chachapoyas (Sonche, Chuquibamba y Chiquilín) y uno en Bagua (Imaza). Sin duda alguna Amazonas se ha convertido en el "nuevo cinturón de pobreza del país. El rostro de la pobreza se refleja 
entre los niños y niñas. La pregunta es si Amazonas es un departamento con vastas potencialidades en turismo, ecología, agropecuaria y cultura ¿por qué no puede dar el gran salto hacia el desarrollo? (Torrejón, 2021).

Por otra parte, se conoce que Amazonas es la región que más población expulsó (emigró) perdió casi el $8 \%$ de su población total. Situación que coincide con el clima de conflictos sociales. La ausencia del Estado, en todas sus instancias, como proveedor de servicios básicos a sus pobladores para aspirar a un nivel de vida mínimamente adecuado ayudaría a explicar tanto la emigración de esta zona de selva como los niveles de protesta y violencia registrados.

Son numerosas las necesidades de capacitación académicas, científicas y sociales que no han sido satisfechas a través de los diversos Programas de formación en ejecución, existen otras insatisfechas o de mayor complejidad, cuya atención reclaman tanto de las instituciones educativas como sociales de su área de influencia. Las instituciones en general deben gestionar su exposición a los riesgos sociales y ambientales mediante el desarrollo de Sistemas de Gestión que incluyan Programas de Educación de diversa índole, a fin de reducir su exposición al riesgo global, regional y local. Son numerosos los casos o ejemplos de colapso de economías que se traducen en mayor pobreza, desocupación, pérdida de infraestructura, etc. que demuestran la vulnerabilidad social y ambiental. Se necesita desarrollar y formar profesionales, así como fortalecer la institucionalidad en el uso de procedimientos, instrumentos de evaluación social y ambiental, y la capacidad interna para detectar y gestionar la resiliencia ante los riesgos que son cada vez más frecuentes e intensos. Los diversos sectores sociales y productivos de los países enfrentan numerosos problemas puntuales $y$, a menudo, monodisciplinarios, que si bien son abordados exitosamente por los profesionales y técnicos; existe, sin embargo, la necesidad de abordar otros problemas más complejos de estos mismos sectores, a través de soluciones creativas y rigurosas.

El objetivo es que los diversos segmentos de la población juvenil sin acceso a la educación superior y capacitación profesional, puedan aprender utilizando las fortalezas de sus entornos cercanos como las instituciones llámese cuarteles. Estas posibilidades son viables por su naturaleza innovadora, pues la idea es "todos ganan" las FF. AA contando con voluntarios para el servicio militar, la universidad con alumnos para formarlos como profesionales y los Municipios con futuros profesionales que contribuyan al desarrollo de sus pueblos. Por otra parte, si queremos que Perú mantenga la identidad de sus pueblos amazónicos, debemos tomar conciencia del valor que representa para la humanidad y evitar que al morir los hijos de nuestros compatriotas indígenas con ellos va muriendo su pueblo y su cultura. Surgió la pregunta ¿de qué vale saber que la Amazonia es una región muy rica en diversidad biológica y cultural, pero con habitantes empobrecidos?

\section{MÉTODO}

La propuesta del modelo se basó en la búsqueda del conocimiento, empleando el método de la etnografía (Gutiérrez, 2011), que utiliza la técnica de la observación científica de los hechos sociales, complementado con mi experiencia y el trabajo empírico con comunidades indígenas que me permiten afirmar que la observación es pieza fundamental para confrontar oportunidades, fortalezas, con mayor razón cuando se trata del encuentro entre comunidades, culturas $y$ racionalidades diferentes como es el caso de las zonas de frontera.

La técnica de la observación es una actividad realizada por un ser vivo (en este caso el ser 
humano), que detecta y asimila el conocimiento de un fenómeno, o el registro de los datos; consiste en la medida y registro de los hechos observables, según el método científico, y, por lo tanto, medida por instrumentos científicos. Además, estas observaciones deben ser realizadas profesionalmente, en la medida de lo posible, sin la influencia de opiniones preconcebidas (Gutiérrez, 2011).

\section{RESULTADOS Y DISCUSIÓN}

\section{Propuesta del Programa}

El Programa Académico de Educación universitaria Inclusiva UniFFAAGoL, es un programa que abre sus puertas y posibilidades de formación y capacitación educativa a todas las personas que desean acceder y participar de esta propuesta bajo la modalidad de convenio con las municipalidades y fuerzas armadas del Perú diseñada para contribuir al desarrollo personal y social de los jóvenes que viven en la zona de frontera y que no tienen posibilidades de adquirir una profesión. El UniFFAAGoL, asume que la educación inclusiva es un símbolo del respeto por toda la humanidad, y constituye la bisagra del cambio en el acceso a la educación que es el respeto a los otros a pesar de la diferencia. Es en esta perspectiva que el UniFFAAGoL, tiene el compromiso de ofertar una formación profesional y capacitación de calidad en su modalidad de convenios con las Municipios y las Fuerzas Armadas del Perú.

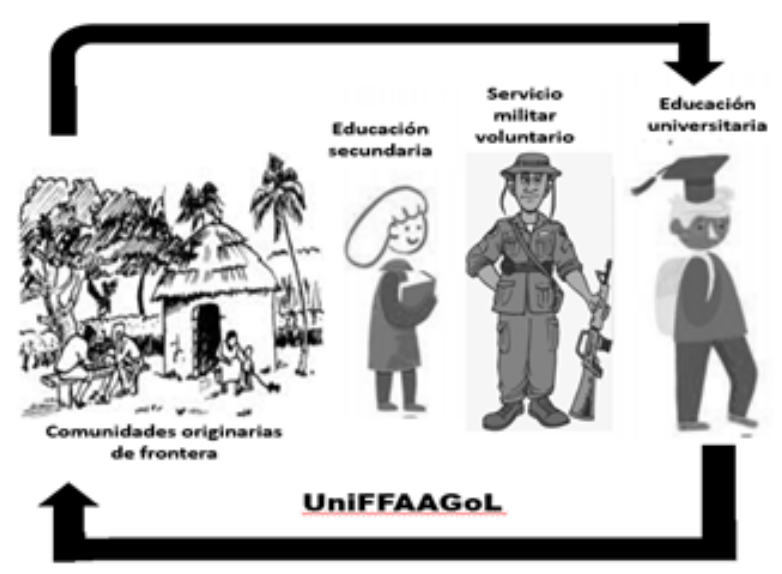

Figura 1. Representación del Programa UniFFAAGoL.

\section{Etapas de formación}

\section{Primera etapa: Servicio militar voluntario $y$ estudios en universidad}

Los interesados ingresan al servicio militar voluntario en los cuarteles fronterizos donde además existen cerca universidades nacionales o privadas donde se someten al examen de admisión correspondiente y optan por carreras principalmente de Ingeniería mecánica, enfermería, administración, contabilidad. Se espera que en esta etapa durante los 2 años que dura el servicio militar desarrollen competencias para el ejercicio de la defensa de la patria y adquieran sentido de responsabilidad, disciplina y cultivo de valores, además se formarán como futuros profesionales en las carreras o especialidades elegidas. El financiamiento en esta etapa lo realizarán las Fuerzas Armadas que brindarán alimentación, hospedaje y propinas. (Figura 2). 


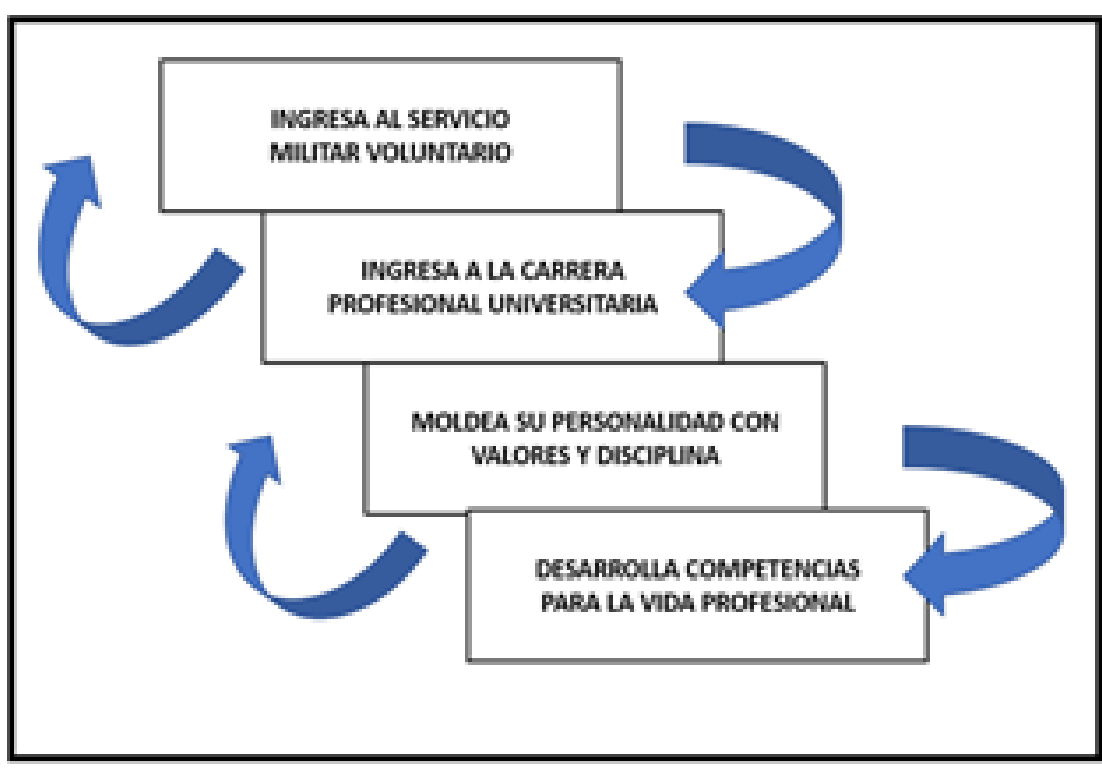

Figura 2. Representación de la primera etapa del programa UniFFAAGoL.

Segunda etapa: Continuación de estudios universitarios vía Beca Municipal

El programa UniFFAAGoL, prevé la continuación de la formación universitaria mediante una beca municipal para los 3 años siguientes que dura los estudios universitarios, el financiamiento estaría a cargo de los Gobiernos Locales. (Ver figura $3)$.

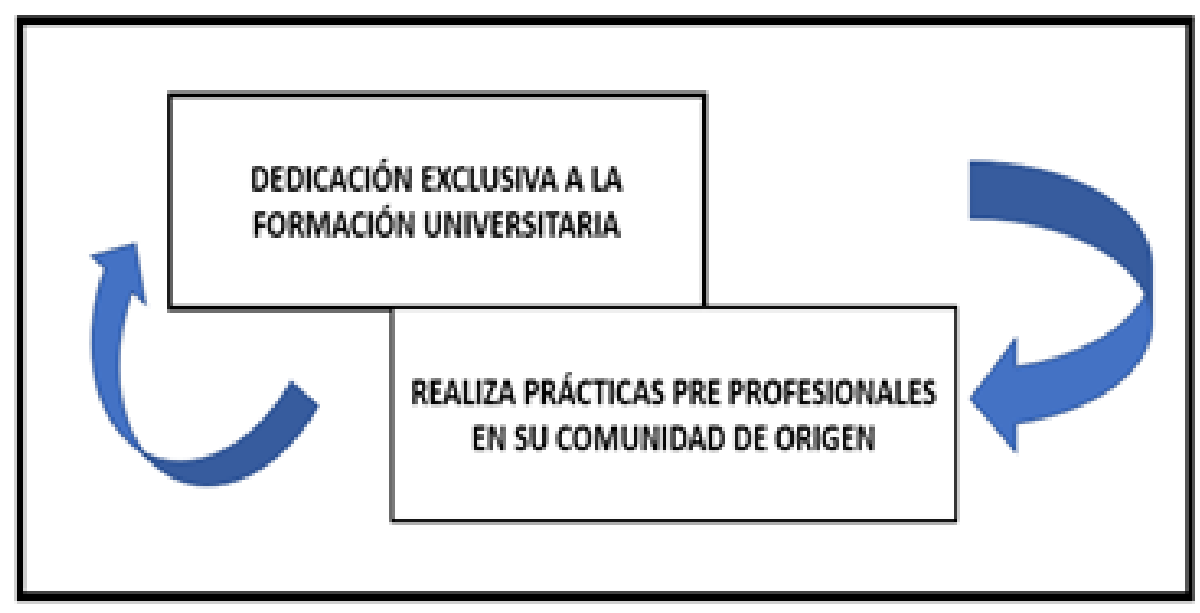

Figura 3. Representación de la segunda etapa del programa UniFFAAGoL

Tercera etapa: Inserción en el campo laboral local

Concluida la formación universitaria, los Gobiernos Locales que financiaron los estudios universitarios preverán plazas para ser ocupadas por los profesionales quienes servirán a sus comunidades como retribución y de esta forma contribuirán al Desarrollo de sus pueblos. (Figuras 4 y 5 ). 


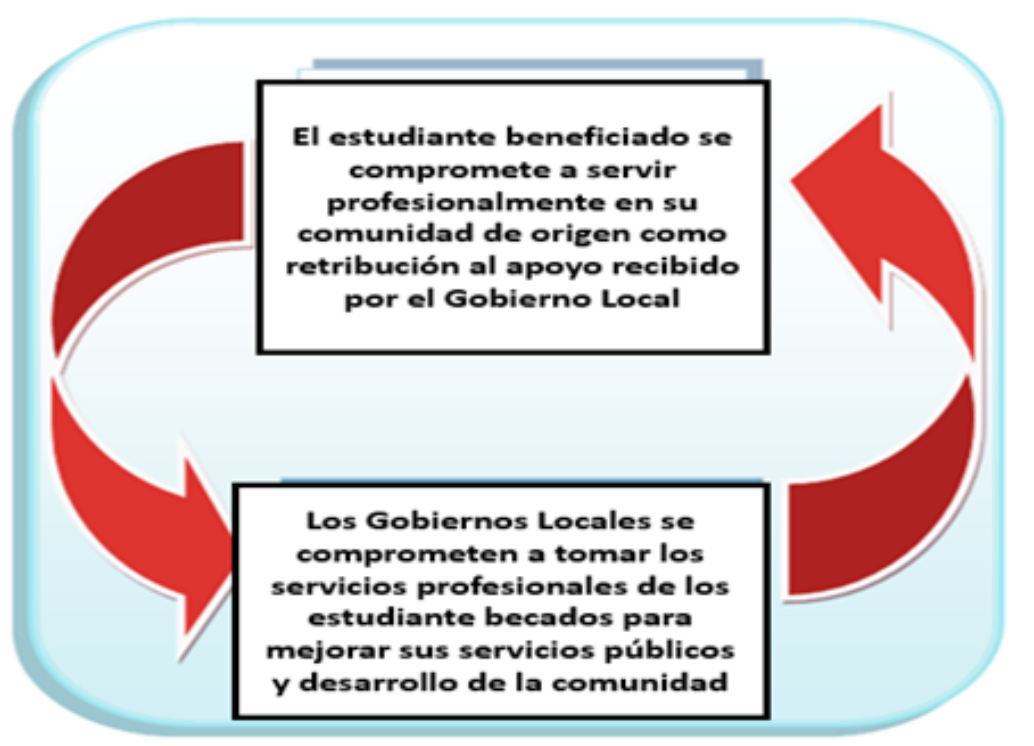

Figura 4. Representación de tercera etapa del programa UniFFAAGoL

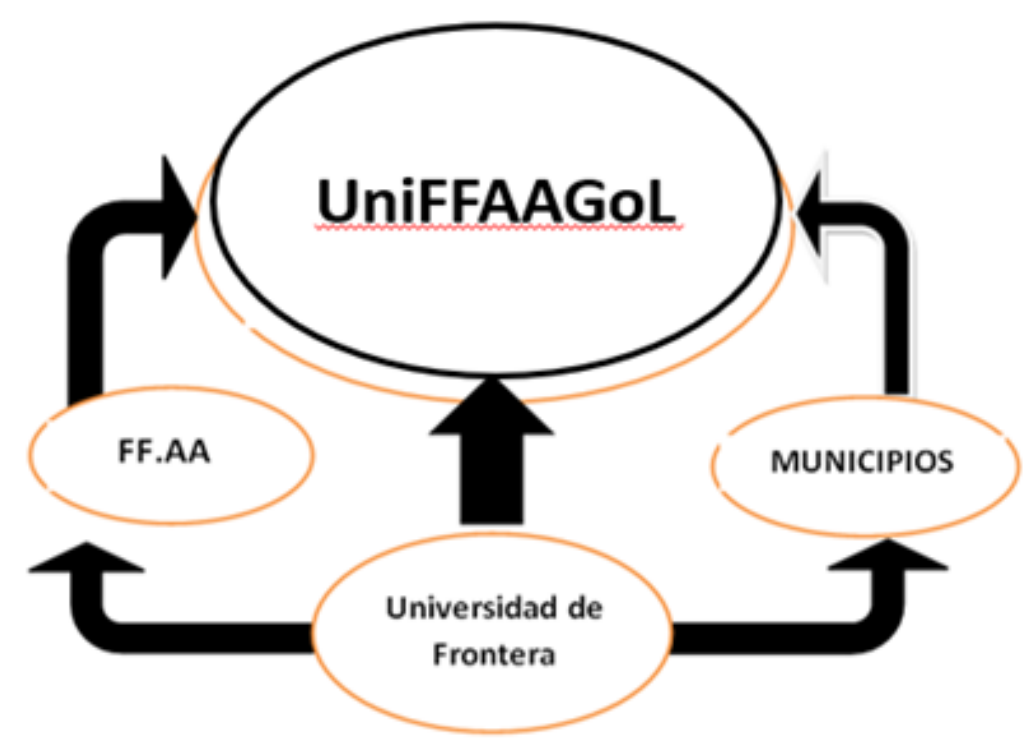

Figura 5. Representación del modelo UniFFAAGoL

Compromisos de las instituciones para la puesta en marcha del UniFFAAGoL

\section{Universidad}

- Selección de postulantes que cumplan con los requisitos exigidos por ley
- Otorgar todas las facilidades para el cumplimiento del servicio militar a los ingresantes a la universidad.

- Mantener informado a las instancias correspondientes sobre el rendimiento de los estudiantes bajo esta modalidad. 


\section{Fuerzas Armadas}

- Otorgar todos los beneficios a los soldados universitarios de acuerdo a Ley. Específicamente otorgarles la propina para sus estudios universitarios durante los 2 años que dure su servicio.

- Dar facilidades a los soldados que ingresan a la universidad, para el cumplimento de sus tareas universitarias.

\section{Gobiernos Locales}

- Otorgar becas a los soldados universitarios al término de su servicio para que puedan completar su formación profesional. Específicamente subvencionar la continuidad de los estudios universitarios por 3 años, asegurando la culminación de los mismos.

- Generar puestos de trabajo en los gobiernos municipales locales.

\section{Discusión}

El Programa se sustenta en paradigmas de naturaleza ontológica, gnoseológica y antropológica, que permite delinear la forma de abordar el estudio de la realidad y define la manera de actuar sobre ella para mejorarla.

Los altos índices de desocupación, delincuencia y pobreza conocidos como desastres sociales, sumados a los últimos acontecimientos de climas extremos naturales, colocan en alto riesgos las economías, vida y sustentabilidad de los pueblos, cuyas instituciones y personal no cuentan con instrumentos, metodologías y procedimientos para reducir su exposición a los riesgos, por lo que se requiere incorporar la componente Educación en zonas de frontera para disminuir la vulnerabilidad y asegurar la sostenibilidad. Actualmente a nivel mundial la pandemia está desnudando las carencias y vulnerables de las economías, de la salud y educación sobre todo las comunidades o pueblos originarios, tal como lo afirma Yvette Sierra (2020), "la situación de salud de los pueblos indígenas por enfermedades infecto-contagiosas introducidas ya es grave debido a su alta prevalencia y el muy deficiente servicio de salud. El coronavirus agravaría más esta situación" (s.n.). Por su parte Baldovino (2020), indica que pese a que la Amazonía ocupa más del 60\% del territorio de Perú y que el 30\% de la población peruana se identificó como indígena en el último censo realizado en el 2017, estamos aún muy lejos de ser un país con una clara política y visión intercultural, que reconozca, respete y sobre todo valore a sus pueblos indígenas, su cultura y la riqueza de nuestro patrimonio cultural. Esta pandemia mostró de la forma más cruel nuestras diferencias y cómo, en casi doscientos años, no hemos logrado asimilar nuestra identidad y promover desarrollo con equidad e inclusión.

En las últimas décadas se viene observando la insuficiencia de efectivos de tropa en los cuarteles el cual es un factor determinante del Ejército, debido a que influye en el cumplimiento de sus funciones operacionales, teniendo en cuenta que actualmente las nuevas tendencias y roles que le han sido incrementados a las Fuerzas Armadas y por ende al Ejército, es combatir las nuevas amenazas que aqueja a Perú (Ministerio de Defensa, 2007). El ejército, para completar sus cuadros, debe desplazar a su personal para captar soldados a lugares alejados de la selva como: Caballococha, Gueppi, Estrecho, Urakuza, entre otros, debido a que los jóvenes de las ciudades que radican aledaño a los cuarteles, muy pocos se presentan para cumplir su obligación con la Nación. Sin duda esto constituye un problema para la defensa nacional, según este autor de 64,000 efectivos que existía en el año 1990, se redujo a 
40,147 en el año 2007 y al año 2017 se captaron 22,305 . El poco interés de los jóvenes por alistarse en el ejército está en las pocas oportunidades que encuentran al terminar su acuartelamiento, hasta la fecha solo se les ofrece acceso vías becas para formación técnica. Esto demuestra que cada vez son menos los jóvenes que viven en zona de frontera que terminan la secundaria y por necesidad de subsistencia se enrolan en las fuerzas armadas, pero al término del servicio militar quedan en su mayoría sin opción de profesionalización y ocupación.

Los tiempos actuales imponen diseñar nuevos modelos y dentro de ellos el de gestión universitaria como una condición absolutamente necesaria para gestar una nueva Universidad que contribuya al Desarrollo de los pueblos originarios. La experiencia de Bocanegra (2012-2013) confirman que si es posible aplicar con éxito esta afirmación.

Los resultados mostrados indican que la globalización como fenómeno mundial nos conduce a romper con el paradigma de que los jóvenes vengan a la universidad, cambiando por otro paradigma como el de que "la universidad vaya hacia los jóvenes". Es indudable que la capacidad de comprender el entorno y de relacionarse activamente con él constituye la señal decisiva para juzgar el grado de autonomía de un sujeto, individual o colectivo. Por lo mismo, el grado de autonomía y pertinencia de la institución universitaria sólo puede juzgarse por referencia al contexto en que opera. La universidad debe direccionar su actuación hacia el acercamiento a la gente, vale decir llevar la universidad a los pueblos que más la necesitan para alcanzar su desarrollo. Un repaso de las visiones y misiones de las diversas universidades de Perú nos muestran que ninguna incorpora la inclusión social (entendida como la real y efectiva oportunidad a jóvenes de comunidades andinas y amazónicas), menos se proyectan como acercar la institución educativa superior a los jóvenes de las zonas más alejadas, es decir se mantiene la tradicional concepción centralista y excluyente. Por isomorfismo podríamos preguntarnos, acaso el soldado no se acerca a su pueblo, y no es del pueblo. Si lo mencionado se acepta como verdad, entonces es hora de asumir el reto de revertirlo y plantear como alternativa de nueva gestión la propuesta de un programa de educación inclusiva.

\section{CONCLUSIONES}

En las zonas de frontera se evidencia problemas urgentes de atención referidos a satisfacción de demanda de profesionales, de soldados acuartelados y de oportunidades para los jóvenes que egresan de la secundaria.

Las universidades actualmente participan limitadamente del proceso de desarrollo en las zonas de frontera, donde existe poco interés de los jóvenes egresados de nivel secundario en acceder a estudios superior por falta de oportunidades, por otra parte, los gobiernos locales carecen de profesionales para mejorar su gestión y los pocos que existen solo permanecen poco tiempo por las distancias y altos costos.

La frontera puede considerarse como ámbito territorial, o espacio local, o hábitat, determinado por ciertos límites. A este ámbito le corresponde un concepto de sociedad local, formada por diversas y máso menosarticuladascomunidades yasociaciones sectoriales, vecinales, étnicas, y los ámbitos de uno o más gobiernos jurisdiccionales, mercados de trabajo, etc. que cuenta con innumerables fortalezas para su Desarrollo. Para cumplir con estos enunciados es requisito desarrollar y establecer nuevas relaciones entre la universidad y el desarrollo de la frontera.

El desarrollo de la frontera, requiere de un nuevo paradigma, basado en un desarrollo económico - social particular, sustentado en su potencial 
endógeno como son los saberes y conocimientos ancestrales fusionados con los conocimientos modernos. Este desarrollo económico - social, pasa por tomar en cuenta las capacidades, habilidades y destrezas productivas, relacionales, comunicacionales, de la iniciativa y creatividad de todos los miembros de esa sociedad local, organizados y capaces de regular sus inevitables conflictos de intereses, interétnicos, ideológicos, políticos, pero compartiendo un proyecto de sociedad más democrática, más igualitaria, más integradora. Como objetivos estratégicos para el desarrollo de la frontera se plantea 1) Saberes e inteligencia intercultural, prospectiva y vigilancia tecnológica, 2) Institucionalidad y gobernabilidad y 3) Desarrollo territorial y sostenibilidad. Estos componentes se recogen en el programa UniFFAAGoL.

\section{REFERENCIAS}

Amilhat-Szary y Rouviére (2009). Des dynamiques transfrontalières au bilan d'aménagement du territoire: innovations et blocages dans les Andes centrales (Chili-Pérou-Bolivie)https:// www.researchgate.net/publication/281955153_ Des_dynamiques_transfrontalieres_au_ bilan_d'amenagement_du_territoire_ innovations_et_blocages_dans_les_Andes_ centrales_Chili-Perou-Bolivie_co-auteur_ Laetitia_Rouviere_32_p_181-196

Baldovino. S.2020.Perú... ¿Y dónde estánlospueblos indígenas? https://www.actualidadambiental. pe/peru-y-donde-estan-los-pueblos-indigenas/

Bocanegra, G.C. (2012). Modelo de Gestión Universitaria Intercultural, Descentralizada con Inclusión Social. Imprenta Gráfica Alemar SAC. Trujillo

Bocanegra, G. C. (2013). Programa de Educación Universitaria Inclusiva para jóvenes que presten servicio militar en zonas de frontera. Partida registral: 005562013. INDECOPI
Gutiérrez, Q. M. (2011). "Método” de investigación etnográfica: observación participante. https://scholar.google.com/citations? user=5AwruR0AAAAJ\&hl=es\#d=gs_md_cita - d\&u=\%2Fcitations\%3Fview_op\%3Dview _citation \% 26hl\%3Des\% 26 user\%3 D 5 A 3D5AwruR0AAAAJ\%26citation_for_view\% 3D5AwruR0AAAAJ\%3 AeQOLeE2rZwMC $\% 26$ tzom $\% 3 \mathrm{D} 300$

King. L y Sabine Schielmann. (2004). El reto de la educación indígena: experiencias y perspectivas.

https://repositorio.minedu.gob.pe/bitstream/ handle/20.500.12799/1845/El\%20 reto $\% 20$ de $\% 201$ a $\% 20$ educacion $\% 20$ indigena $\% 20$ experiencias $\% 20$ y $\% 20$ perspectivas. pdf? sequence $=1$

Ministerio de Defensa, 2007. Compendio estadístico del sector defensa. https://www.mindef.gob. pe/informacion/documentos/Compendio_ Estadistico_2005-2007.pdf

Ministerio de Salud. (2019). Programa de entrenamiento en salud pública dirigido a personal del servicio militar voluntario. https:// repositorio.ins.gob.pe/xmlui/bitstream/handle/ INS/1140/Programa_entrenamiento_Salud_ Publica_Unidades_Facilitador_Tomo_1. pdf? sequence $=1 \&$ isAllowed $=y$

PRONABEC. (2016). Programa beca 18 y pueblos indígenas diagnóstico región loreto - perú http://d2g1dofgkbpvym.cloudfront.net/2016/1 0/21/15/14/02/414/2016.10.DIAGNOSTICO_ BECA_18.VFINAL

Torrejón, P (2021). La justicia y equidad, un reto del Bicentenario. http: // elmertorrejonpizarro. $\mathrm{com} /$.

UNESCO. (2004). El Reto de la educación indígena: experiencias y perspectivas http://unesdoc. unesco.org/images/0013/001347/134774s.pdf

UNESCO (2019). Cultura y desarrollo sostenible. https://es.unesco.org/system/files/private_ documents/informe_-onu_-2019.pdf 
Sierra, Y. 2020. Pueblos indígenas: Los más vulnerables frente al avance del Coronavirus en América Latina. https:/es.mongabay. com/2020/03/pueblos-indigenas-coronavirusamerica-latina/
Zapata-Barrero, Ricard y Ferrer-Gallardo, Xavier (eds.) (2012) Fronteras en movimiento: Migraciones hacia la Unión Europea en el contexto Mediterráneo. Barcelona: Ediciones Bellaterra, 352 p. ISBN: 978-84-7290-574-0 